\title{
Radiation Oncology: Contouring for Patients with Primary Breast Cancer for
}

\section{Clinical Protocol Compliance}

\author{
Anna Shlimak1 | Maureen Britton ${ }^{1}$ | Maryann Bishop-Jodoin ${ }^{1,2}$ | Linda Ding 1 | Carla Bradford ${ }^{1}$ | \\ Harry Bushe $^{1}$ | Abdulnasser Khalifei ${ }^{1}$ | Fenghong Liu ${ }^{1}$ Kenneth Ulin ${ }^{1,2}$ | Yankua Fan ${ }^{1}$ | I-Lin Kuo ${ }^{1}$ | \\ John G. Roubil ${ }^{1}$ | Matthew Iandoli1,2 | Suhong Yu1 | Jonathan Saleeby ${ }^{1}$ | Eric Ko ${ }^{1}$ | Daniel Han ${ }^{1}$ | \\ Janaki Moni $^{1}$ | Allison Sacher ${ }^{1}$ | M. Giulia Cicchetti ${ }^{1}$ | Shirin Sioshansi ${ }^{1}$ | Beth Herrick ${ }^{1}$ | Jonathan \\ Glanzman $^{1}$ | Lakshimi Shanmugham ${ }^{1}$ | Thomas Quinn ${ }^{1}$ | Shannon Higgins ${ }^{1}$ | Julie Trifone ${ }^{1}$ | Joshua \\ Taylor $^{1}$ | Marious Croos ${ }^{1}$ | Bashera Nochomowitz ${ }^{1}$ | Linda Taylor ${ }^{1}$ | Karen Morano ${ }^{1,2}$ | Kathleen \\ Briggs $^{1,2}$ | Shawn Kirby ${ }^{1,2}$ | Jean Quigley ${ }^{1,2}$ | Heather Reifler ${ }^{1,2}$ | Fran Laurie $^{1,2}$ | Amanda Ignacio ${ }^{1,2}$ | \\ Katherine Karolczuk $^{1,2}$ | TJ FitzGerald ${ }^{1,2}$
}

*Correspondence: TJ FitzGerald

Address: ${ }^{1}$ Department of Radiation Oncology, University of Massachusetts Medical School, Worcester, MA; ${ }^{2}$ Imaging and Radiation Oncology Core (IROC) and the Quality Assurance Review Center, Lincoln RI e-mail $\bowtie:$ TJ.FitzGerald@umassmemorial.org

Received: 08 July 2021; Accepted: 19 July 2021

Copyright: (C) 2021 Shlimak A. This is an open-access article distributed under the terms of the Creative Commons Attribution License, which permits unrestricted use, distribution, and reproduction in any medium, provided that the original work is properly cited.

\section{ABSTRACT}

Radiation therapy is an important tool in the care of patients with breast cancer. Today's breast cancer clinical trials demonstrate the volumetric-based strategies for contouring breast and regional lymph node targets and normal tissue can be common threads as well as points of differentiation. In this paper, we review approaches for contouring targets in breast cancer clinical trials to help site investigators effectively treat targets and meet normal tissue constraints.

Keywords: Radiation Therapy, Breast Cancer, Volumetric-Based Treatment, Normal Tissue Contraints, Contouring Targets

\section{Introduction}

Radiation therapy for patients with primary breast cancer has significantly changed over the past 50 years. Prior to the development of computed tomography (CT) based volumetric planning, patients were simulated for treatment using fluoroscopy calculated with two-dimensional geometry and isodose distribution only generated through the isocenter of therapy. If nodal regions in the supraclavicular region and axilla were included in the therapy plan, matched non divergent field edges were aligned using multiple techniques to create a match line between the fields treating the breast/chest wall and nodal regions in a single therapy plan. The breast contour was taken one plane at in the region of the breast isocenter and compensation devices for the slope of the medial and lateral breast were placed to 
accommodate the slope of the chest wall in this single plane. The results for tumor control were excellent and equivalent to mastectomy, therefore radiation therapy was established as an adjunct to breast preserving surgery for primary breast cancer management as well as an invaluable tool in treating multiple clinical situations for this disease (Fisher et al., 2002; Veronesi U et al., 1981; Sarrazin et al., 1989; Blichert-Toft et al., 1992; van Dongen et al., 2000; Jacobson et al., 1995).

Although the technique had merit and made good use of available technology at the time, there was no optimal accurate method to capture volume information for both tumor associated targets and normal tissue in the therapy field. Effort was made to use historical data for injury to optimize planning for radiation therapy, however volumes had to be estimated and accurate conclusions for dose volume analysis could not be firmly established from previous data.

Simulation with three-dimensional planning based using CT simulation changed the paradigm of radiation oncology. Radiation oncologists had to pivot and alter work flow. Although most generational radiation oncologists were not trained with CT-based planning, these tools were applied to planning and radiation oncologists adjusted the planning process and applied volumetric metrics for daily work. Radiation oncologists had to learn how to contour and draw the breast, surgical cavity, and draining lymph nodes on CT with tools designed for a new generation. Institutions had varied approaches to contouring and were asymmetric in the process of both acquiring and applying tools for daily work, therefore there was non-uniformity in the adoption of contouring tools and volumetric metrics in the radiation oncology community which led to asymmetry in the application of volumetric planning. Individual departments worked to develop guidelines however these remained confined to department use and often varied greatly between departments and even colleagues treating similar patients in the same department.

Volumetric guidelines and normal tissue constraints in breast cancer clinical trials became a common thread between departments willing to participate on trials involving radiation therapy. Important questions in breast cancer management relative to radiation therapy targeting, dose, and fractionation can only be effectively answered today within the context of a clinical trial as these often require significant accrual objectives to answer study questions. Likewise, it is important for participants in clinical trials to apply radiation therapy objects in as uniform a manner as possible to ensure that the results of the trial can be accurate and can be applied successfully into daily clinical management.

A good series of examples of clinical trials that addressed issues associated with breast cancer care were conducted by the Cancer and Leukemia Group B (CALGB) beginning in 1993 evaluating compression of chemotherapy cycles with Cytoxan/Adriamycin with the addition of Taxol in node 
positive patients (Henderson et al., 2003; Citron et al., 2003). Because radiation therapy was not thought to influence survival, information concerning radiation therapy was not collected on study. In 1997, information became available that radiation therapy had a positive impact on patient survival in patients with node positive breast cancer and the importance of radiation therapy in this disease was both established and vetted (Morris et al., 1997). In these series of studies conducted by the CALGB, more than 3,000 patients were accrued on trial. An attempt was made to review radiation therapy data by retrospectively acquiring treatment information including radiation dose and volume treated to the breast, chest wall, and regional lymph nodes, however data could only be collected in approximately $30 \%$ of patients as many institutions and institutional review board (IRB) members concluded that patients were going to require a secondary consent to permit data transfer for objects not intended to be transferred as part of the primary study. Of those patients whose data could be collected, the radiation dose and volume treated were extremely varied and no conclusion could be drawn relative to the dose volume effect to breast, chest wall, and regional lymph nodes. However, there was a tendency for patients treated with Taxol to also have been treated with radiation therapy. The important point is that without radiation therapy guidelines written into the study and a process for establishing quality assurance that the guidelines were followed, dose/ volume issues were inconsistent, and no conclusions could be drawn relative to radiation dose volume matters and control of disease (Sartor et al., 2005).

This issue continued into more modern trials. The American College of Surgeons Oncology Group Z0011 (ACOSOG Z0011) Alliance trial was designed to evaluate therapeutic titration to the axilla in patients with breast cancer including patients with limited and no axillary intervention. Radiation therapy was intended to be delivered just to the breast without intentional extension of therapy into lymph node draining targets. Radiation therapy data was not collected on study and no real time quality assurance was performed to confirm target placement and intended dose (Giuliano et al., 2017). In retrospective analysis of data collected after completion of the study, it was found that many high-risk patients did have intentional radiation therapy directed to lymph node drainage regions, thus complicating interpretation of the study and the application of radiation therapy in the non-protocol community setting (Jagsi et al., 2014). These examples demonstrate the non-uniform application of contouring objects for breast cancer care and at both a department and clinical trial level, can lead to asymmetry of object application for breast cancer care. This has become more difficult in the era of hypofractionation and sub-total volume breast cancer radiotherapy care.

Non-uniform application of contouring objects for breast cancer care remains a challenge for assigning volumetric metrics for breast radiation therapy and patient care, especially in management of patients on study. There is inconsistency in contouring the breast, chest wall, and regional lymph node 
draining regions among well trained radiation oncologists and significant variety in radiation therapy treatment planning techniques. Although modern technology including volume modulated arc therapy can limit regions of dose inhomogeneity within a target, often unmodulated advanced technology can push unintentional and potentially toxic dose to structures including cardio-pulmonary parenchyma and contralateral thorax/chest wall despite homogeneous distribution of therapy within the intended tumor target. National Clinical Trial Network (NCTN) breast cancer protocols assign normal tissue constraints for dose volume homogeneity for multiple structures (Emami et al., 1991). Radiation oncologists are often gifted problem solvers, however problem-solving can often be at the expense of the assigned normal tissue constraints.

In this paper, we review strategies for contouring targets required for breast cancer radiation therapy to help investigators treat targets effectively and meet normal tissue constraints assigned for protocol management. This may help us answer important questions moving forward for radiation therapy treatment planning and optimal application of contouring strategies for breast cancer clinical trials.

\section{Principles of Volume-based Therapy}

Contouring breast volumes should be done with both the anticipation and understanding by the involved radiation oncologist what the plan should resemble when complete. This is essential to optimize department work flow, otherwise throughput and work flow can be significantly disrupted in the daily practice of radiation therapy.

Prior to the 2014 consolidation of the National Cancer Institute's (NCI) National Cooperative Groups, the Radiation Therapy Oncology Group (RTOG) created a breast contouring atlas building consensus with outstanding investigators in the design and contouring tissue targets (White et al (no date)). The intent of the project was not designed to develop a radiation therapy treatment plan to meet protocol guidelines for target and normal tissue constraints, however the atlas is referenced in many breast cancer protocols as the primary source for contouring targets. This brings both strength and weakness. The strengths are identified as providing a framework for target design and permits site investigators a pathway to contour in a manner to similar to experts in our field. The weakness is the atlas does not have a plan associated with the contours and this creates controversy in defining targets and generating plans to meet protocol constraints. Each case in the atlas has idealized geometry not uniformly seen in daily clinical practice, which presents a challenge for site investigators who want to apply the strategy associated with the atlas to real world clinical practice and protocol management. 
Normal tissue constraints are imbedded in breast cancer clinical trials and metrics for study deviations are written into each study based on the ability of site investigators to adhere to normal tissue guidelines. The categories for case evaluation include the case being acceptable, variation acceptable, and deviation unacceptable based on guidelines. Excessive dose to one normal tissue structure will cause the case to be scored as deviation unacceptable. Although metrics can vary between studies, current objectives include the following for patients being treated to the breast and/or chest wall and regional lymph node draining areas. The total lung dose volume maximum is 20 Gy to $20 \%$ of total lung volume. The pulmonary volume on the affected side is limited to 20 Gy to $35 \%$ of the lung in patients receiving regional radiation therapy and $30 \%$ to patients being treated to the intact breast. The mean heart dose must be less than 4 Gy with variation acceptable at 5 Gy. Ten (10) Gy is the limit to the contralateral breast and/or chest wall in selected studies. Each of these constraints bring challenges to the planning team. The constraints are likewise under constant revision. As our technology improves including optical tracking and breath hold, normal tissue constraints are being further titrated to optimize dose to normal tissue. Depending on the geometry and chest wall separation, the target dose can be made uniform often at the expense of pushing dose into normal tissue of concern including heart, lung, and contralateral chest wall. Likewise, protocols often set limitations for radiation dose uniformity in treatment targets, therefore the planning team must balance constraints between target and normal tissue to meet study objectives. In this paper, we review strategies for contouring targets in breast cancer protocols to help site investigators achieve study objectives and balance normal tissue and target constraints required by the NCI and the Cancer Therapeutic Evaluation Program (CTEP).

\section{Contouring the Breast}

Numerous two, three, and four-dimensional techniques have been developed by radiation oncologists and institutions over the past 50 years for the delivery of radiation therapy for breast cancer patients. Institutions will often support multiple techniques of therapy driven in large part by the clinical assessment of different radiation oncologists within each institution. This alone can create challenges for both physics/dosimetry and therapy teams especially today when it becomes increasingly more difficult to support multiple computer programs and therapy pathways within a single department. Fluoroscopicbased planning generally had the radiation oncologist place a marker at the chest wall midline and at a lateral position defining breast tissue in the therapy position, often situated at or slightly anterior to the posterior axillary line. Today, often radiation oncologists continue to apply this practice when they lack absolute confidence in their ability to contour breast tissue from CT. Although often accurate and reproducible for daily care, adjustments in position were largely influenced by the perception of lung volume potentially incorporated into the therapy field based on judgment on fluoroscopy seen by the 
radiation oncologist. Therefore, before the advent of CT planning, there was only limited information concerning the volume of tissue treated and computational analytics were limited to radiation dose distribution at the level of the isocenter, often based on orthogonal images.

CT planning created a new era in radiation oncology treatment delivery. The volume of tissue potentially treated could be calculated and the volume of radiation dose distribution could be reviewed in detail including volume metrics for radiation dose asymmetry. Technology quickly matured including daily image guidance insuring that dose/volume distribution in tissues could be replicated daily with confidence. Areas of dose to both intended therapy targets and normal tissue could be analyzed with dose/volume histograms and compared for outcome analysis. Dosimetry can be measured by portal images to validate daily treatment execution. Radiation oncologists, however, were largely trained using fluoroscopy for treatment planning, therefore adjustments to volumetric planning were challenging. With fluoroscopy, a large amount of physician work was accomplished in the simulator. Using volumetric planning, the physician effort only began when the imaging was completed and could be transferred to work stations for contouring with computer tools unfamiliar to mid and late career physicians. Physicians sought consistency in contouring, hence the reference to atlases and directives provided by disease experts.

On review of the RTOG axial atlas, the breast volumes are drawn to midline and extend posteriorly to the latissimus muscle in the lateral plane. This is helpful at multiple levels and the Children's Oncology Group (COG) Hodgkin lymphoma protocols apply the volumes drawn in the atlas to assess risk of developing breast cancer relative to the fields of radiation therapy. If, however, radiation therapy plans were developed with the contours representing planning target volume to the breast, in many patients it would be challenging to meet constraints to lung, heart, and contralateral breast and provide full coverage of tumor associated targets as drawn. To bring $100 \%$ of prescription dose to midline often requires significant extension of dose into the contralateral breast. Likewise, treating a left-sided patient to a breast target drawn to the latissimus would include a significant portion of heart and lung in the therapy field. Contouring the breast to midline and drawing target posteriorly to muscle makes it difficult to meet normal tissue constraints and homogeneity guidelines through the breast target defined in the atlas. As can be seen in Fig 1, contouring to midline and the latissimus results in high dose volume histogram to ipsilateral and total lung, heart, and contralateral breast. To meet constraints, drawing breast tissue alone permits dose to be distributed to the breast as intended but also permits less dose to normal tissue. This is the natural extension of dose as the planners place a constraint in the underlying heart and lung and dose will naturally be "pushed" in the medial and lateral plane and provide dose to tissue in these locations independent of contour. Fig 2 is the same patient with breast tissue contoured 
without exaggerated contouring to midline or the latissimus. Note the dose distribution to target and the improvement in dose volume histogram analysis to normal tissue by this change in contour.

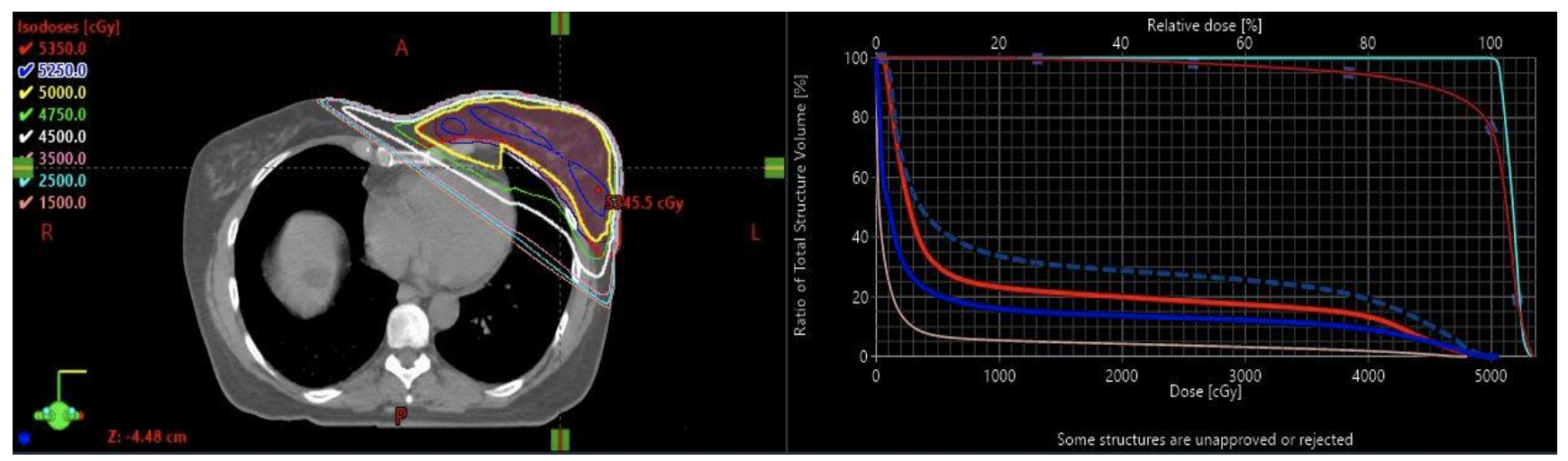

Figure 1: RTOG Atlas-derived contouring to midline and the latissimus results in higher dose to ipsilateral and total lung, heart, and contralateral breast. The cyan line represents the cavity, dark red is breast volume, dashed blue is total lung, bright red is heart, and pink is contralateral breast. Courtesy of the Department of Radiation Oncology, University of Massachusetts Medical School and White et al., (2018).

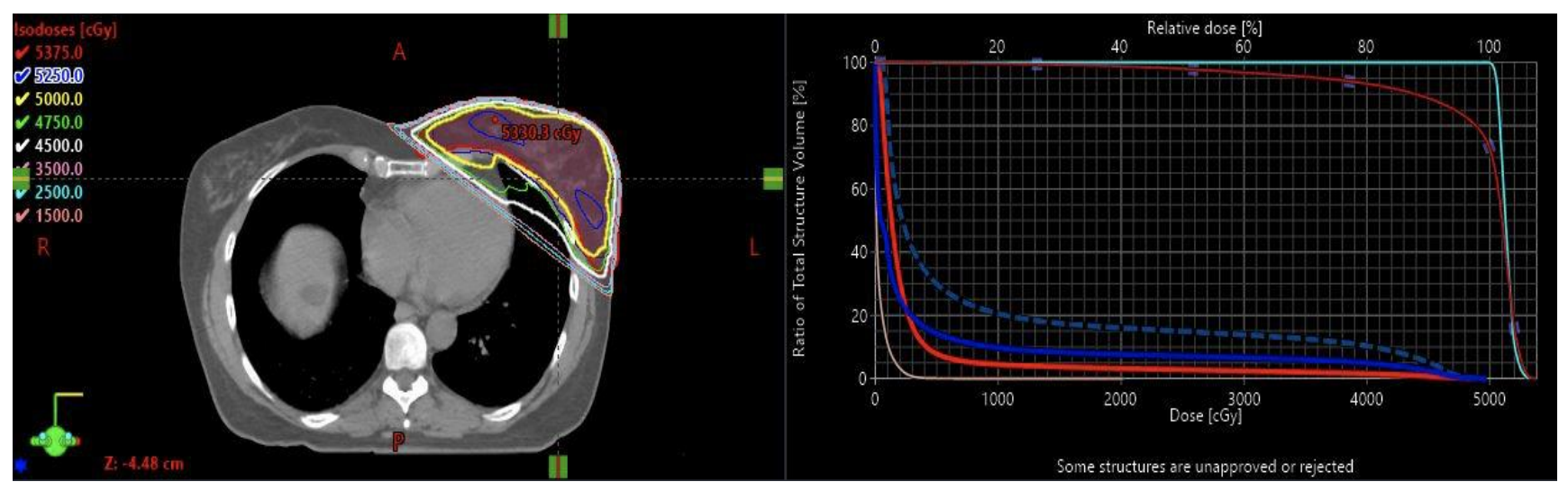

Figure 2: Same patient with breast tissue contoured without exaggerated contouring to midline or the latissimus.

The cyan line represents the cavity, dark red is breast volume, dashed blue is total lung, bright red is heart, and pink is contralateral breast. Courtesy of the Department of Radiation Oncology, University of Massachusetts Medical School and White et al (no date).

Fig 3 and Fig. 4 provide a similar example in a different patient as to how much a difference can be seen in dose volume analysis to normal tissue when breast contouring is limited to breast tissue without exaggerated contouring to midline and the latissimus. Contouring in this manner will help all study investigators meet protocol objectives for dose volume management of tissue and normal tissue targets including the heart, lung parenchyma, and contralateral breast. While limiting dose to normal tissue structures can increase dose inhomogeneity across the target, planning optimization can help mitigate this point including mixed energy plans when needed. 


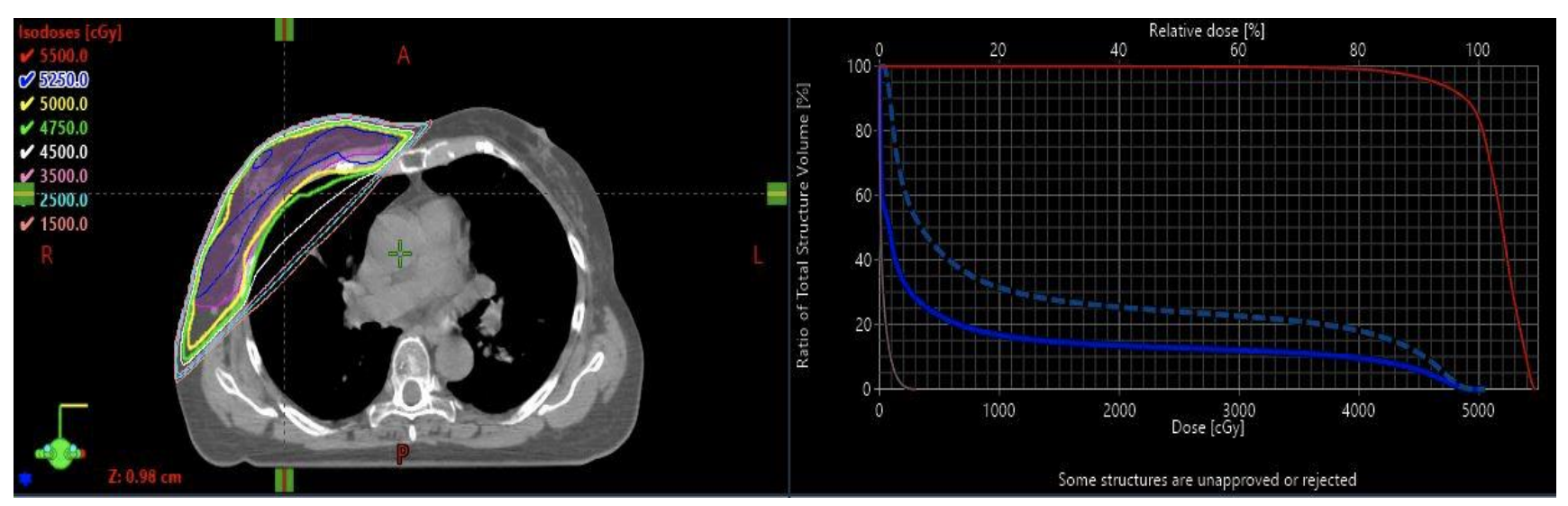

Figure 3: A similar example of RTOG Atlas-derived contouring (in a different patient) as to how much a difference can be seen in dose volume analysis to normal tissue when breast contouring is limited to breast tissue without exaggerated contouring to midline and the latissimus (Figure 4). In Figure 3, dark red is breast volume, dashed blue is total lung, solid blue is right lung, and pink is contralateral breast. Courtesy of the Department of Radiation Oncology, University of Massachusetts Medical School and White et al., (2018).

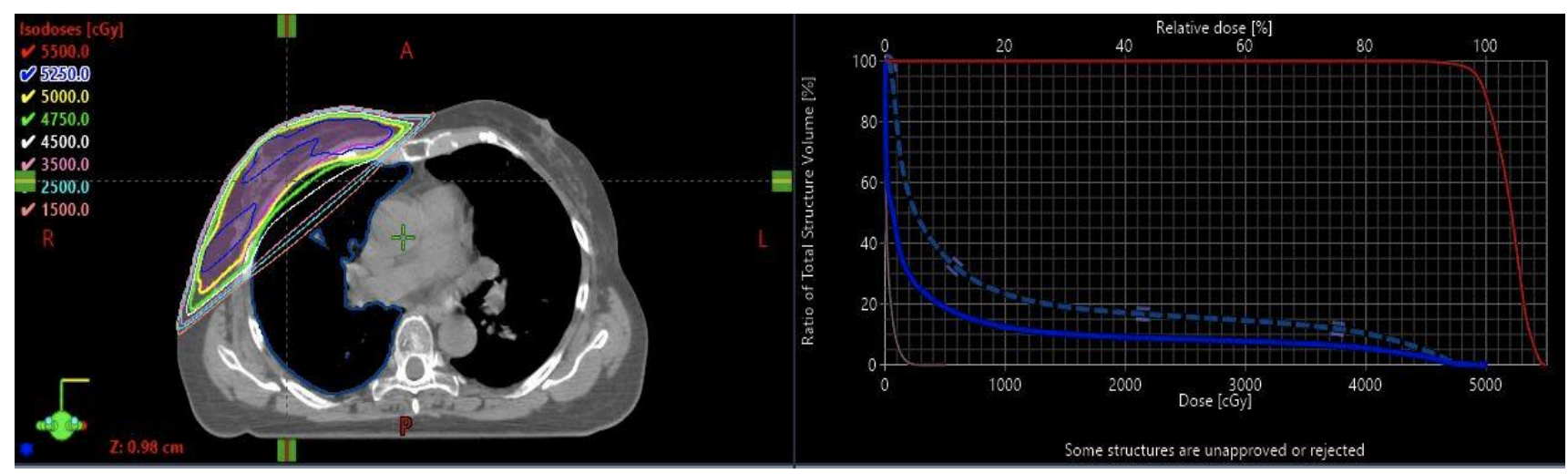

Figure 4: Same patient as in Figure 3, contoured with less exaggerated breast volume. Note the difference in dose to

lung. In Figure 4, dark red is breast volume, dashed blue is total lung, solid blue is right lung, and pink is contralateral breast. Courtesy of the Department of Radiation Oncology, University of Massachusetts Medical School and White et al (no date).

\section{Contouring the Internal Mammary System}

Most investigators recognize that the internal mammary lymph nodes do not lend easily to definition as a volume since the lymph system is symbiotic and parallel to the internal mammary artery. Most investigators contour the artery on each axial CT slice and reconstruct the lymph node region as a longitudinal structure slightly lateral to the sternum along the artery as it is the only structure visible on planning computer tomography. Breast cancer protocols with patients with known positive axillary lymph nodes will often require intentional therapy directed to the internal mammary structure. This circumstance can also create planning issues similar to contouring the breast and chest wall to midline, and by default, often a compromise must be reached to balance dose to target and meet normal tissue 
constraints. To acknowledge this point, modern protocols often ask for $80 \%$ of the prescription dose be delivered to the internal mammary system with assigned variation acceptable as a compromise to meet normal tissue objectives, otherwise efforts to achieve protocol dose would be at the expense of normal tissue constraints. Fig. 5 and Fig. 6 provide an example with dose volume histogram analysis of two plans on the same patient with intentional and unintentional coverage of the internal mammary system and the associated results on dose volume histogram analysis between the two plans.

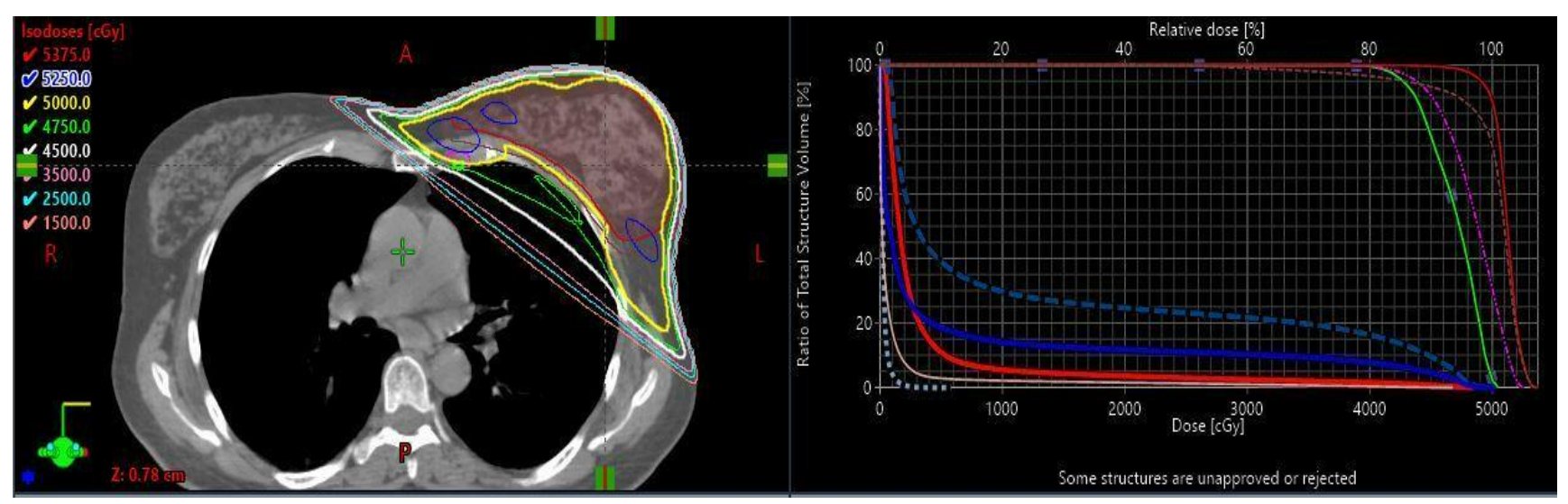

Figure 5: An example with dose volume histogram analysis of two plans on the same patient with intentional coverage of the internal mammary system and the associated results. In Figure 5, intentional coverage of internal mammary nodes (IMNs), the heart is bright red, the total lung is dashed blue, the left lung is solid blue, the contralateral breast is pink, the IMN clinical target volume (CTV) is solid green, the IMN PTV is purple broken, the breast CTV is dark red, and the breast planning target volume (PTV) is brown dashed. Courtesy of the Department of Radiation Oncology, University of Massachusetts Medical School.

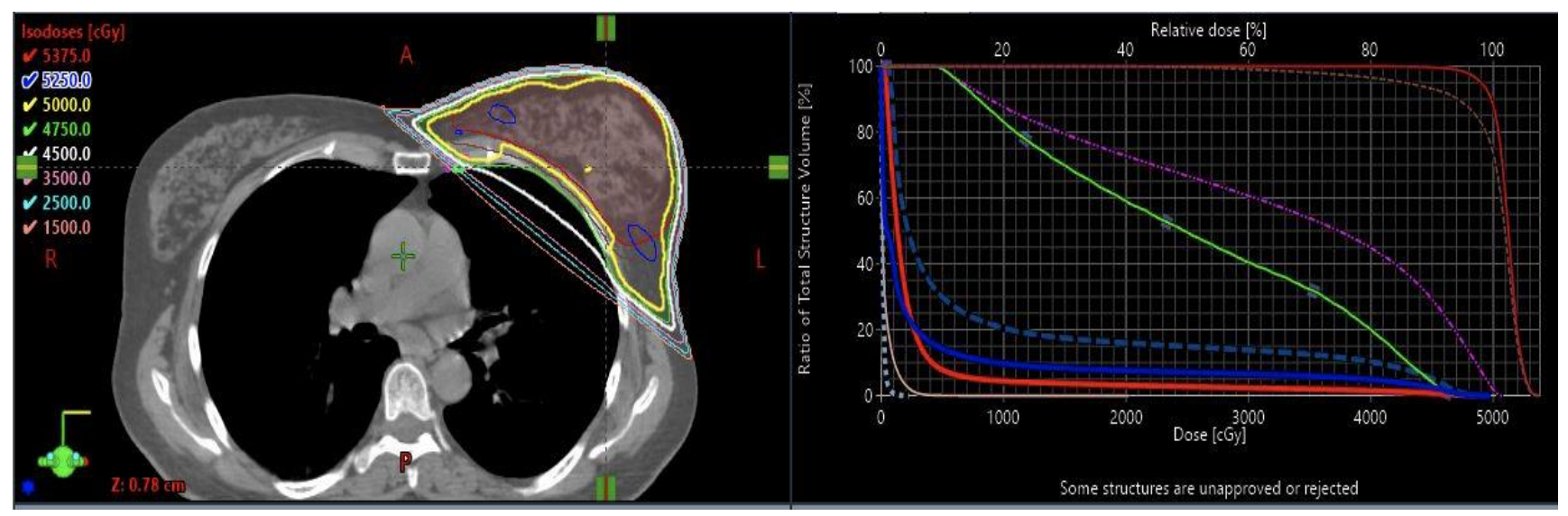

Figure 6: An example with dose volume histogram analysis on the same patient with unintentional coverage of the internal mammary system and the associated results on dose volume histogram analysis between the two plans. In Figure 6, the heart is bright red, the total lung is dashed blue, the left lung is solid blue, the contralateral breast is pink, the IMN CTV is solid green, the IMN PTV is purple broken, the breast CTV is dark red, and the breast PTV is brown dashed. Courtesy of the Department of Radiation Oncology, University of Massachusetts Medical School. 
An additional consideration is drawing the target into the medial supraclavicular region. As can be seen on Fig. 7, the lymph node drainage of the breast, although thought to be compartmentalized, is largely interconnected in the lateral, superior, and medial segments through multiple channels including the intercostal system (Wikimedia Commons, 2006). The RTOG atlas depicts the superior extent of the internal mammary chain at approximately the first intercostal space and contours the structure as an isolated compartment. This leaves a gap in contour between the superior internal mammary target and the medial supraclavicular region. Planning engines simply want to please us and will not place dose in areas not contoured, therefore with patients entered on recent studies, this region at the level of the head of the clavicle has been under contoured, hence underdosed or cold. Historically without volumetric objects, the irony is this area would have been in the match line using two-dimensional planning tools, therefore possibly receiving $140 \%$ of prescription dose over non-divergent matched fields (Siddon et al., 1983). Many study investigators on modern studies prefer the internal mammary volume drawn as a continuum into the medial supraclavicular region as opposed to two separate independent compartments when regional volumes are included as the strategy of therapy. An example of the contour and location along the sternum in the chest wall is seen in Fig. 8 with contiguity to the medial supraclavicular region. Investigators evaluating SPECT/CT imaging have demonstrated the importance of the first intercostal space as a site of interest for the internal mammary system supporting the strategy to draw the medial supraclavicular area and the internal mammary system as a continuum or a continuous volume (Wang et al., 2020).

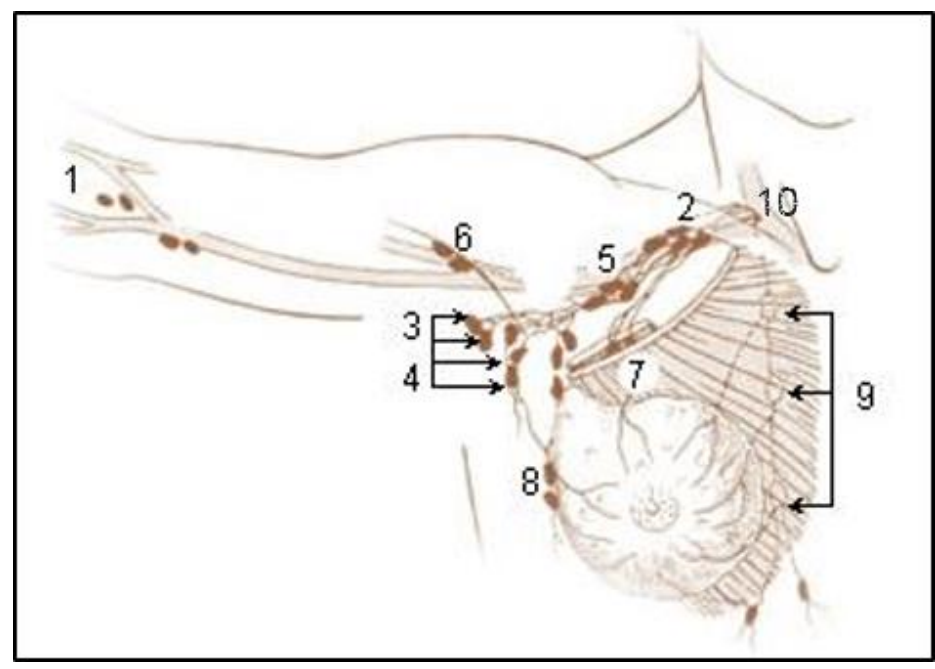

Figure 7: Lymphatic drainage of the breast. Note the continuity of the internal mammary to the supraclavicular lymph node chains. 1. Cubital; 2. Apical axillary; 3 \& 4. Lateral (surface) axillary; 5. Central axillary; 6. Brachial axillary; 7. Interpectoral; 8. Paramammary; 9. Parasternal (internal mammary); 10. Supraclavicular. Adapted from A labelled diagram of some of the lymph nodes of the arm and the pectoral region [Online]. Available at: https://commons.wikimedia.org/wiki/File:Illu_lymph_chain03.jpg. (Accessed 07 July 2021). 


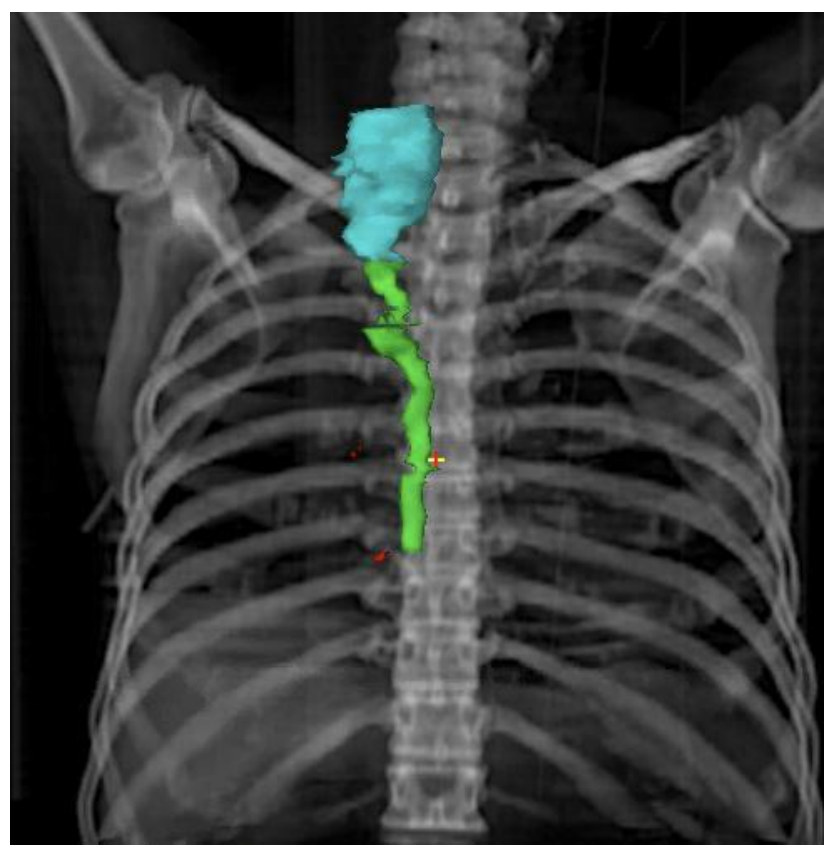

Figure 8: Most study investigators on modern studies prefer the internal mammary volume drawn as a continuum into the medial supraclavicular region as opposed to two separate compartments. In this figure, the supraclavicular volume is blue, the IMN volume is green. Courtesy of the Department of Radiation Oncology, University of Massachusetts Medical School.

\section{Contouring Axillary and Supraclavicular Lymph Node Regions}

Contouring lymph node regions can be a challenge as important clinical information identified for each patient will influence the volume and extent of contouring and the target volumes treated can be patient specific. The extent of surgery including axillary dissection and skeletonization of the axillary vessels and findings on pathology will influence both radiation dose/volume and outcome. There has been movement in the past 10 years to limit the extent of axillary surgery and place more emphasis on comprehensive radiation management of regional lymph node draining regions in protocol management (Donker et al., 2020). Previous investigations have suggested that atlases may be helpful for initial target definition, however clinical information may require investigators to individualize contours to patient specific situations, especially in situations of extracapsular spread of disease into soft tissues including the presence of residual disease along vessel and nerve structures that could not be removed without high risk of injury. Investigators have suggested that areas of concern for extended axillary disease may not be fully represented in the atlas and have advocated for more extended fields than drawn.

Most investigators define levels 1 and 2 of the axilla relative to the pectoralis minor muscle with the superior edge defined where the axillary vessels cross the muscle (Fig. 9). Lymph nodes lateral and inferior to the muscle are generally considered level 1 and lymph nodes inferior and superior to the 
muscle are considered level 2. Level 3 nodes are generally considered medial and superior to level 2 and reside at and around the clavicle and sub-clavicular regions (Fig. 9). Most of the clinical impression of the axilla felt on physical examination is level 1 and, not without exception, most often sentinel lymph node biopsies are in level 1. Without axillary dissection, more of these nodal groups are more easily visualized on radiation oncology planning imaging as these nodes remain in situ undisturbed by surgical change. These are represented by Fig. 10.

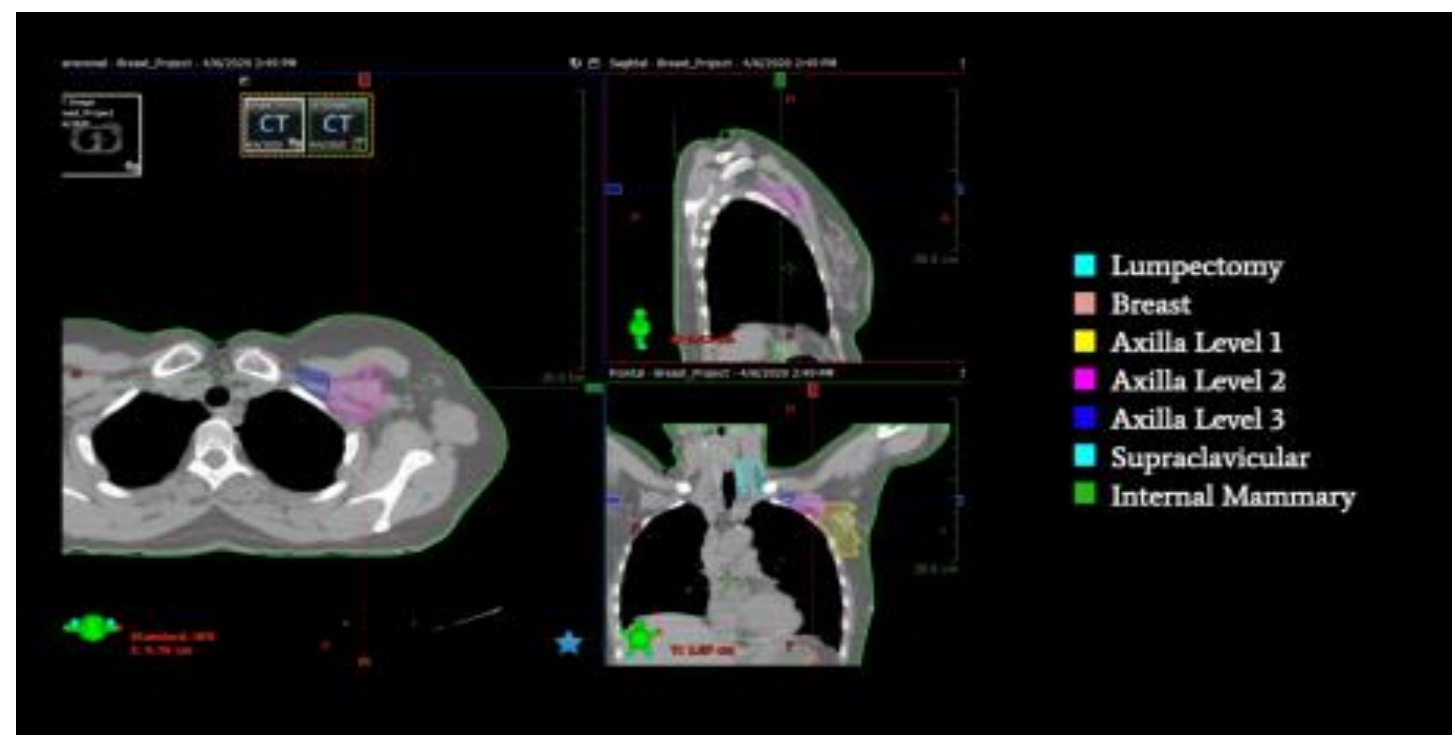

Figure 9: Levels 1 and 2 of the axilla relative to the pectoralis minor muscle with the superior edge defined where the axillary vessels cross the muscle. Courtesy of the Department of Radiation Oncology, University of Massachusetts Medical School.

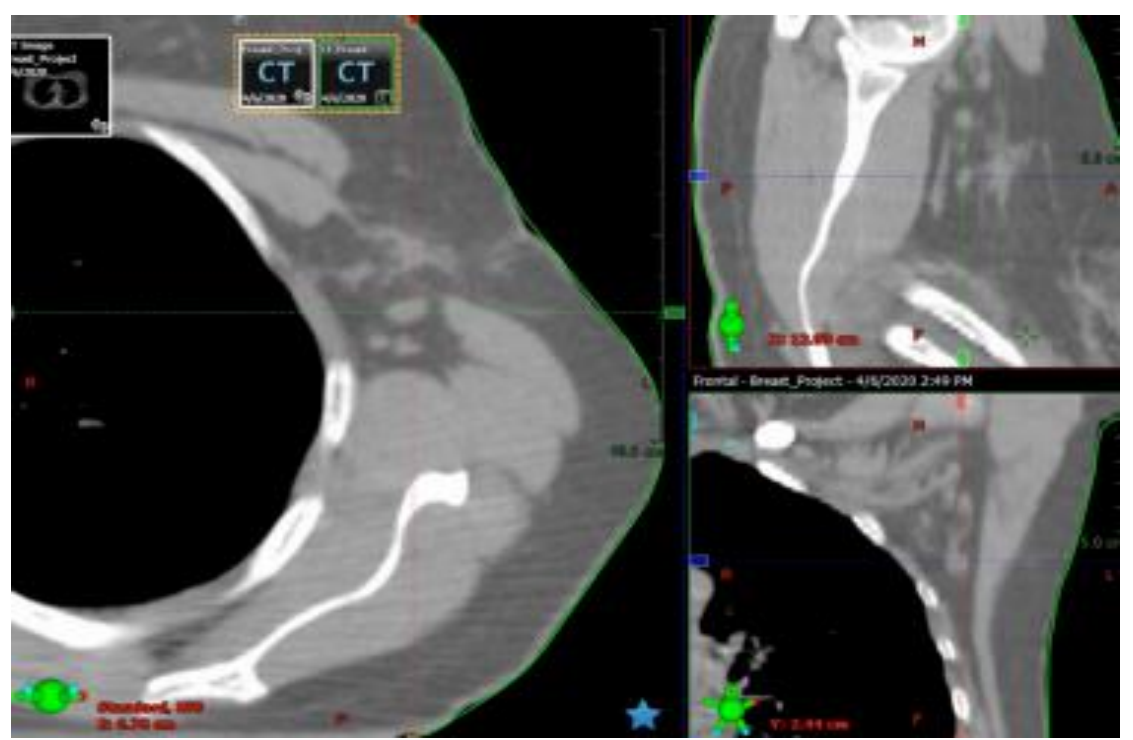

Figure 10: Axillary nodes seen on planning scan. Without axillary dissection, more of these nodal groups are more easily visualized on radiation oncology planning imaging as these nodes remain in situ undisturbed by surgical change. Courtesy of the Department of Radiation Oncology, University of Massachusetts Medical School. 
Attempts have been made by radiation oncologists to titrate the lateral and posterior extent of lymph node contours in patients that have more extended surgery and axillary dissection. Historically, if patients had extended axillary surgery, radiation oncologists would limit the lateral extent of the supraclavicular volume at the parallax of the coracoid process with extension to include a portion of the humeral head in patients who had more limited surgery and required more comprehensive nodal irradiation. Many radiation oncologists likewise would not intentionally use a posterior field and assume anterior fields would achieve the primary objective. As can be seen in Fig. 11, the nodal volumes in the region of the lateral aspect of the clavicle can be at the anterior/ posterior axial midline, therefore planned fields with volumes drawn to midplane can optimize coverage in this location. It has been established that tangential radiation therapy directed to the breast unintentionally treats $60-80 \%$ of level 1-2 axillary tissue depending on what the radiation oncologist chooses as the superior field edge (high/low tangents) (Reznick et al., 2005). Although the ACOSOG Z011 trial suggested that tangential radiation therapy to the breast is enough to treat nodal volumes, review of supplemental information from site investigators reveals that many high-risk patients on study had comprehensive nodal radiation therapy including treatment to the supraclavicular region, thus suggesting the importance of quality assurance on study (Giuliano et al., 2017; Jagsi et al., 2014). Therefore, despite considerable effort by many outstanding study investigators, asymmetry remains in contouring and defining nodal volumes to treat. On study, the recommendations are to treat the supraclavicular fossa to a level inferior to the cricoid and extend the field laterally to either the coracoid for more limited volume therapy or beyond the humeral head with shielding to accommodate the more lateral extension of potential disease in level 1. A study evaluating practice patterns of German investigators confirmed the asymmetric application of nodal contours by radiation oncologists (Borm et al., 2019; Borm et al., 2020).

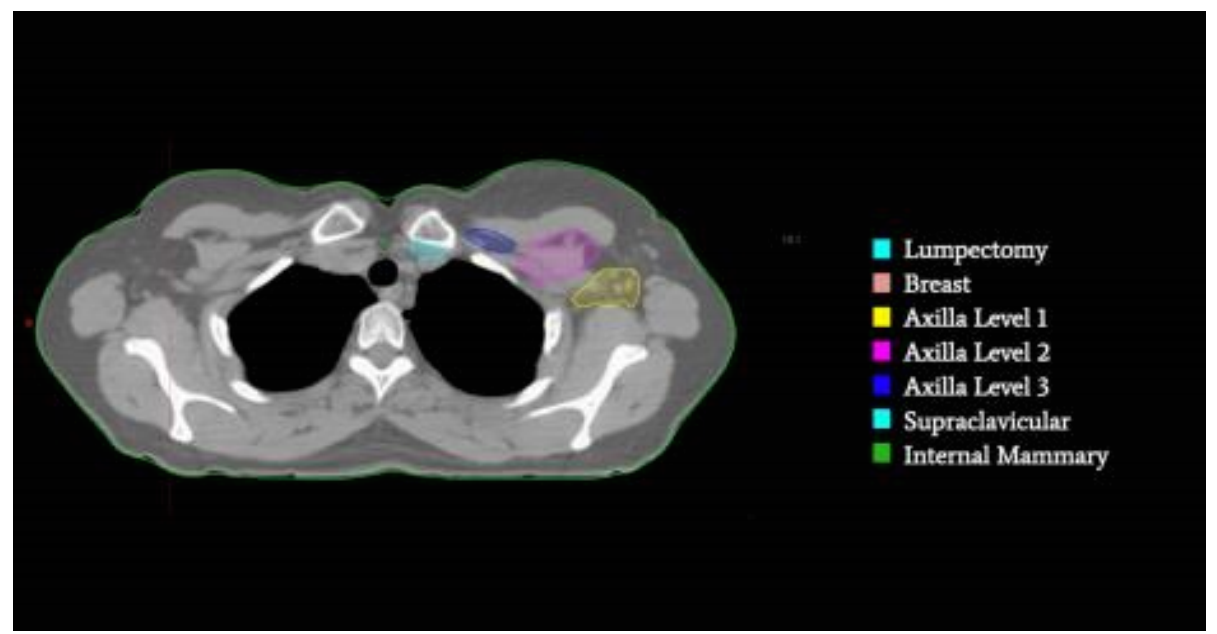

Figure 11: The nodal volumes in the region of the lateral aspect of the clavicle can be at the anterior/ posterior axial midline, therefore planned fields with volumes drawn to midplane can optimize coverage in this location. Courtesy of the Department of Radiation Oncology, University of Massachusetts Medical School. 
Investigators have worked to determine if the contours drawn for nodal volumes meet what is needed for clinical care. A retrospective series was published in 2015 evaluating the atlas relative to disease extension in the axilla in node positive patients. In this series of patients, many node positive patients had evidence of disease extension beyond the contour limits defined by the atlas (Gentile et al., 2015). In fairness, these patients had CT evidence of disease in the axillary region that most radiation oncologists would have likely contoured as part of primary management, nevertheless the important aspect of the review of the objects is to make certain radiation oncologists adjust targets to patient specific details of management.

\section{Conclusions}

While digital atlases help define a contouring strategy of nodal and breast volumes for radiation therapy breast cancer management, it can be challenging to uniformly adapt the contours displayed in atlases and meet target and normal tissue constraints defined in national clinical trials. Targets drawn in atlases often reflect best geometry. Breast cancer radiation oncology planning and management remains challenging for all involved as irregular topography and asymmetric geometry make dose optimization difficult coupled with intentional asymmetry of dose distribution in targets imposed by normal tissue constraints. To meet the challenges generated by normal tissue constraints, compromises must be met in contouring to place dose where needed and limit dose to normal tissue. In this paper, strategies were outlined to help support target coverage and meeting normal tissue constraints defined by studies. In breast cancer contouring, dose will naturally extend to medial and lateral chest wall segments as constraints are applied to the underlying cardiac and pulmonary structures. Although there are times when contours for both breast and surgical cavity must extend to either midline or the latissimus region, this does not have to be standard, otherwise there will be exaggerated areas of dose asymmetry in the medial and lateral breast contour and meeting protocol driven normal tissue constraints will be difficult if not impossible for the planning team. These can be optimized based on patient and protocol specific needs. Nodal volumes can extend to the region of the latissimus in level 1, however clinical judgment can be assigned to coverage and contour needs in this location. If there were nodal disease in this location with extra nodal spread, extending the targets in this location may be necessary in selected circumstances to limit the risk of regional failure. It is difficult at times to bring dose to the internal mammary region to protocol or microscopic dose and meet study constraints, therefore study investigators have also had to compromise protocol objectives and redefine the limits of what would be defined as variation acceptable on study. Many investigators have chosen $80 \%$ of prescription dose as a compromise in finding balance between internal mammary coverage and normal tissue constraints.

Radiation therapy has an invaluable role in the care of patients with breast cancer. The radiation 
oncology community has adopted varied approaches to contouring breast and regional lymph node targets with more consistency applied to contouring normal tissue. The local and regional control rates remain exceptional; therefore, it will be difficult to identify statistical separation to nuances in radiation therapy technique and volume definition as targeting and applied compromise to targets will be driven in large part by normal tissue constraints. It will be important in the design and execution of breast cancer protocols involving radiation therapy to define volumes and acquire radiation therapy objects and correlate them to failure patterns. This will help modify technique and adapt technique and volumes to patient specific situations. If objects and failure patterns were acquired and collated for previous breast cancer, artificial intelligence programs could be applied to the information to correlate disease location, tumor burden, and volume to develop predictive indices for patterns of failure and optimize planning strategies for radiation therapy dose and volume. The future remains bright for the discipline; and optimizing the application of radiation therapy dose and volume for the best outcomes in patient care continues.

\section{References}

Blichert-Toft M, Rose C, Andersen JA, Overgaard M, Axelsson CK, Andersen KW, Mouridsen HT. Danish randomized trial comparing breast conservation therapy with mastectomy: six years of life- table analysis. Danish Breast Cancer Cooperative Group. J Natl Cancer Inst Monogr 1992; 11: 19-25.

Borm KJ, Kessel K, Devecka M, Muench S, Straube C, Schiller K, Schüttrumpf L, Dapper H, Wöller B, Pigorsch S, Combs SE. Variability in lymph node irradiation in patients with breast cancer-results from a multi-center study in German-speaking countries. Strahlentherapie Onkologie 2020; 196: 15-22.

Borm KJ, Voppichler J, Düsberg M, Oechsner M, Vag T, Weber W, Combs SE, Duma MN. FDG/PET CT-based lymph node atlas in breast cancer patients. Int J Radiat Oncol Biol Phys 2019: 103: 574-582.

Citron ML, Berry DA, Cirrincione C, Hudis C, Winer EP, Gradishar WJ, Davidson NE, Martino S, Livingston R, Ingle JN, Perez EA. Randomized trial of dose-dense versus conventionally scheduled and sequential versus concurrent combination chemotherapy as postoperative adjuvant treatment of node positive primary breast cancer: first report of Intergroup Trial C9741/Cancer and Leukemia Group B Trial 9741. J Clin Oncol 2003; 21: 1431-1439.

Donker M, van Tienhoven G, Straver ME, Meijnen P, van de Velde CJ, Mansel RE, Cataliotti L, Westenberg AH, Klinkenbijl JH, Orzalesi L, Bouma WH. Radiotherapy or surgery of the axilla after a positive sentinel node in breast cancer (EORTC 1098122023 AMAROS): A randomised, multicentre, open-label, phase 3 non-inferiority trial. Lancet Oncol 2014; 15: 1303-1310.

Emami B, Lyman J, Brown A, Cola L, Goitein M, Munzenrider JE, Shank B, Solin LJ, Wesson M. Tolerance of normal tissue of therapeutic irradiation. Int J Radiat Oncol Biol Phys 1991; 21: 109-122.

Fisher B, Anderson S, Bryant J, Margolese RG, Deutsch M, Fisher ER, Jeong JH, Wolmark N. Twenty-year follow-up of a randomized trial comparing total mastectomy, lumpectomy, and lumpectomy plus irradiation for the treatment of invasive breast cancer. N Engl J Med 2002; 347: 1233-1241.

Gentile MS, Usman AA, Neuschler EI, Sathiaseelan V, Hayes JP, Small Jr W. Contouring guidelines for the axillary lymph nodes for the delivery of radiation therapy in breast cancer: Evaluation of the RTOG Breast Cancer Atlas. Int J Radiat Oncol Biol Phys 2015; 93: 257-265. 
Giuliano AE, Ballman KV, McCall L, Beitsch PD, Brennan MB, Kelemen PR, Ollila DW, Hansen NM, Whitworth PW, Blumencranz PW, Leitch AM. Effect of axillary dissection vs no axillary dissection on 10-year overall survival among women with invasive breast cancer and sentinel node metastasis: The ACOSOG Z0011 (Alliance) randomized clinical trial. J Am Med Assoc 2017; 318: 918-926.

Henderson IC, Berry DA, Demetri GD, Cirrincione CT, Goldstein LJ, Martino S, Ingle JN, Cooper MR, Hayes DF, Tkaczuk KH, Fleming G. Improved outcomes from adding sequential Paclitaxel but not from escalating Doxorubicin dose in an adjuvant chemotherapy regimen for patients with node-positive primary breast cancer. J Clin Oncol 2003; 21: 976-983.

Jacobson JA, Danforth DN, Cowan KH, d'Angelo T, Steinberg SM, Pierce L, Lippman ME, Lichter AS, Glatstein E, Okunieff P. Ten-year results of a comparison of conservation with mastectomy in the treatment of stage I and II breast cancer. $N$ Engl $J$ Med 1995; 332: 907-911.

Jagsi R, Chadha M, Moni J, Ballman K, Laurie F, Buchholz TA, Giuliano A, Haffty BG. Radiation field design in the ACOSOG Z0011 (Alliance) trial. J Clin Oncol 2014; 32: 3600-3606.

Morris AD, Morris RD, Wilson JF, White J, Steinberg S, Okunieff P, Arriagada R, Le MG, Blichert-Toft M, Van Dongen JA. Breast-conserving therapy vs mastectomy in early stage breast cancer: a meta-analysis of 10 -year survival. Cancer J Sci Am 1997; 3: $06-12$.

Reznik J, Cicchetti MG, Degaspe B, Fitzgerald TJ. Analysis of axillary coverage during tangential radiation therapy to the breast. Int J Radiat Oncol Biol Phys 2005; 61: 163-168.

Sarrazin D, Le MG, Arriagada R, Contesso G, Fontaine F, Spielmann M, Rochard F, Le Chevalier TH, Lacour J. Ten-year results of a randomized trial comparing a conservative treatment to mastectomy in early breast cancer. Radiother Oncol 1989; 14: 177-184.

Sartor CI, Peterson BL, Woolf S, FitzGerald TJ, Laurie F, Turrisi AJ, Bogart J, Henderson IC, Norton L. Effect of addition of adjuvant paclitaxel on radiotherapy delivery and locoregional control of node-positive breast cancer: Cancer and Leukemia Group B 9344. J Clin Oncol 2005; 23: 30-40.

Siddon RL, Buck BA, Harris JR, Svensson GK. Three-field technique for breast irradiation using tangential field corner blocks. Int J Radiat Oncol Biol Phys 1983; 9: 583-588.

van Dongen JA, Voogd AC, Fentiman IS, Legrand C, Sylvester RJ, Tong D, van der Schueren E, Helle PA, van Zijl K, Bartelink H. Long-term results of a randomized trial comparing breast-conserving therapy with mastectomy: European Organization for Research and Treatment of Cancer 10801 trial. J Natl Cancer Inst Monogr 2000; 92: 1143-1150.

Veronesi U, Saccozzi R, Del Vecchio M, Banfi A, Clemente C, De Lena M, Gallus G, Greco M, Luini A, Marubini E, Muscolino G. Comparing radical mastectomy with quadrantectomy, axillary dissection, and radiotherapy in patients with small cancers of the breast. N Engl J Med 1981; 305: 06-11.

Wang X, Wang W, Li JB, Huo ZW, Xu M, Qiu PF, Zhang YJ, Li FX, Wang JZ. Definition of internal mammary node target volume based on the position of the internal mammary sentinel lymph nodes presented on SPECT/CT fusion images. Front Oncol 2020; 9: 1553.

White J, Tai A, Arthur D, Buchholz T, MacDonald S, Marks L, Pierce L, Recht A, Rabinovitch R, Taghian A, Vicini F, Woodward W, Li XL. Breast Cancer Atlas for Radiation Therapy Planning: Consensus Definitions. Available at: Click Here. (Accessed: July 2020).

Wikimedia Commons (2006) A labelled diagram of some of the lymph nodes of the arm and the pectoral region [Online]. Available at: Click Here (Accessed 07 July 2021). 